\title{
"Prevalence of MYD88 L265P mutation in Diffuse Large B-Cell Lymphomas involving the skin: Single-center study in Argentina.".
}

Dana Kohan, Federico Jauk, Julieła Pandolfi, Vicłoria Volonteri, Victoria Ołero, Hernán García Rivello. Pathology Department, litalian Hospital of Buenos Aires, Argentine poor prognosis and shorter overall survival. Aim: Determine the prevalence of MYD88 L265P mutation in PCLBCL-NOS,

\section{Materials and Methods}

Introduction: Cutaneous involvement by diffuse large B-cell lymphoma (DLBCL) can occur primarily in the skin, or secondarily to a systemic DLBCL (S-DLBCL). In the first group, two different clinicopathological entities can be separated: primary cutaneous large B-cell lymphoma not otherwise specified (PCLBCL-NOS), and primary cutaneous large B-cell lymphoma leg-type (PCLBCL-LT). MYD88 L265P mutation was proven to be present in $16,5 \%$ of systemic DLBCL and $40-68 \%$ of PCLBCL-LT cases, and is associated with PCLBCL-LT and S-DLBCL.

A retrospective observational study was performed.

All cutaneous DLBCL cases diagnosed in Italian Hospital of Buenos Aires (Argentina) between 2007 and 2017 were included. 15 cases were
selected and reclassified based

on the WHO criteria (2017) in

three groups: S-DLBCL $(n=6)$,

PCLBCL-NOS $(n=6) \quad$ and PCLBCL-LT $(n=3)$.

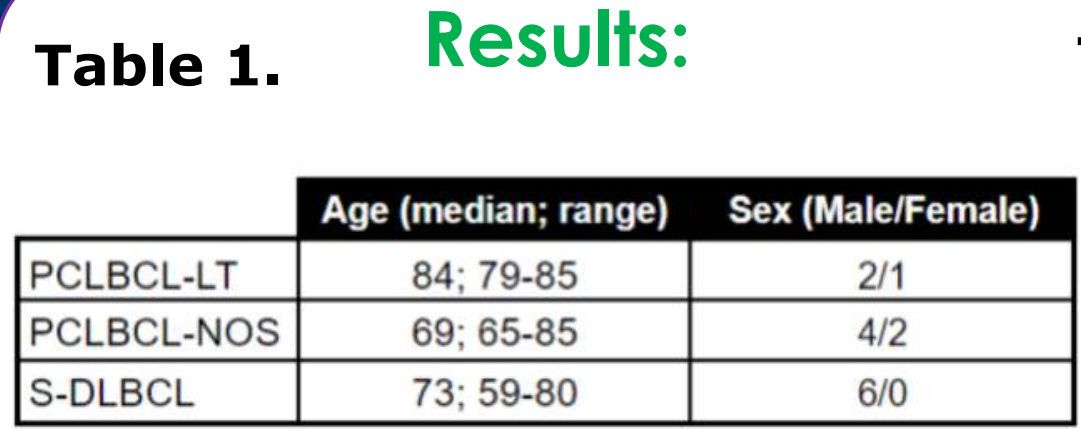

Table 1. Clinical features of the three groups of patients.

Table 2. Location, immunophenotypic profile and MYD88 status in all patients involved in this study.

If necessary to reclassify cases following the WHO (2017) criteria, immunohistochemistry (IHC) was performed, using an automated processor (Benchmark XT, Ventana).

DNA was extracted from formalin-fixed paraffin-embedded tissue, using QIAamp DNA mini kit (QIAGEN).

The presence of MYD88 L265P was assessed using qBiomarker Somatic Mutation PCR Assay Kit, specific for C.794T>C substitution in MYD88 gene.
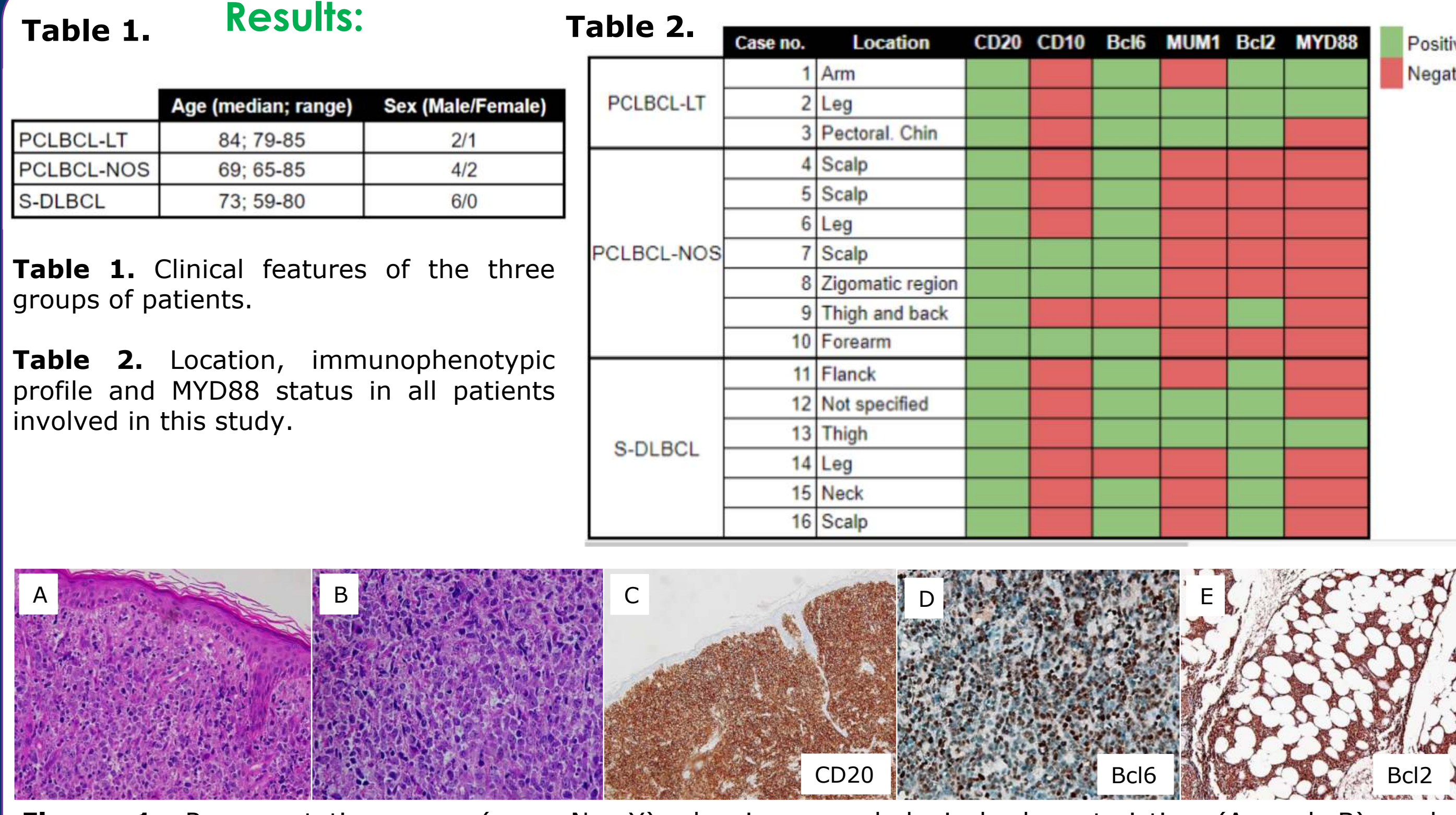

Figure 1. Representative case (Case $N O \quad X$ ) showing morphological characteristics ( $A$ and $B$ ) and immunohistochemical profile of group PCLBCL-LT (C, D and E).

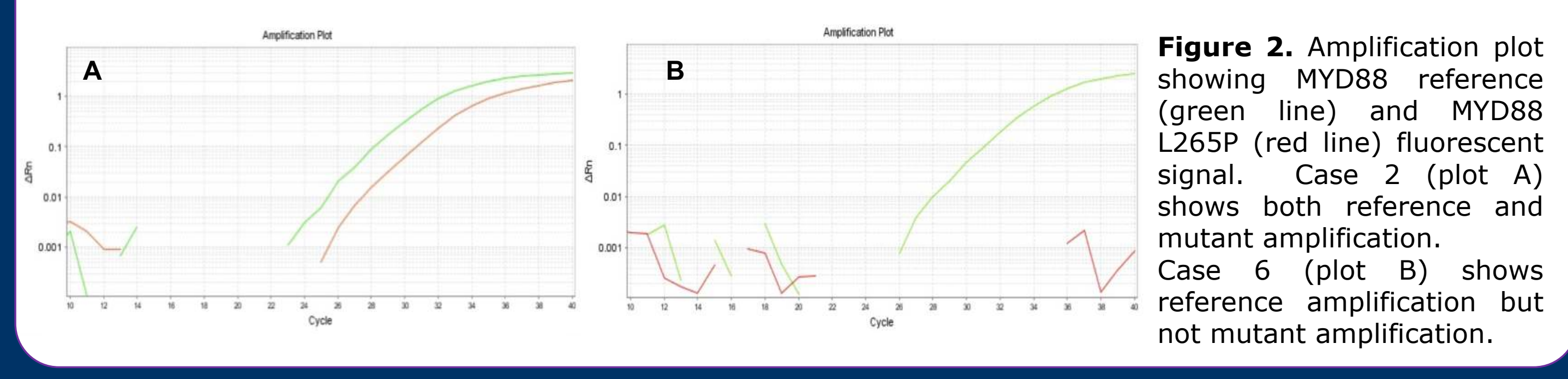

\section{Conclusions}

In our series, L265P MYD88 mutation was observed in $66,7 \%$ of PCLBCL-LT and $16,5 \%$ of S-DLBCL, in coincidence with previous reports.

Although a small amount of cases were included, we plan to expand this study in a collaborative way with other institutions from our country.

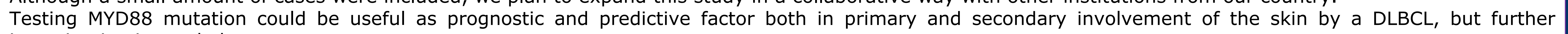
investigation is needed. 